\title{
El aporte social del proyecto de vinculación y la relación con la universidad de los niños
}

\section{The social contribution of the bonding project and the relationship with the children's university}

Norma Garcés Garcés

Universidad de Guayaquil, Ecuador

Zila Esteves Fajardo

Universidad de Guayaquil, Ecuador

Carlos Valle Navarro

Universidad de Guayaquil, Ecuador

Lidia Patricia Estrella Asencio

Universidad de Guayaquil, Ecuador

Autor para correspondencia: norma.garcesg@ug.edu.ec

Fecha de recepción: 23 de agosto de 2018 - Fecha de aceptación: 02 octubre de 2018

\begin{abstract}
Resumen
El artículo es una demostración teórica del valor que tiene el conocimiento de la universidad de los niños en la vida estudiantil, debiendo vislumbrar los diferentes momentos de la relación estudiante- universidad. En la toma de decisiones de los adolescentes con la finalidad de llegar a establecer cómo el conocimiento de la función de la universidad puede mejorar el desempeño de los estudiantes de los primeros años de la universidad como parte de la comunicación interna que los centros de educación superior deben mejorar.
\end{abstract}

Palabras Claves: cognición; universidad; relación; comunicación interna

\begin{abstract}
The article is a theoretical demonstration of the value of the knowledge of the University of Children in student life, having to glimpse the different moments of the student-university relationship. In decision-making of adolescents in order to establish how knowledge of the role of the university can improve the performance of the students of the first years of the university as part of the internal communication that higher education centers they must improve.
\end{abstract}

Key Words: cognition; university; relationship; internal communication 


\section{Introducción}

La temática que se investiga da inicio con una orientación vocacional y ayuda para que el estudiante al momento de decidir o elegir estudiar una profesión que luego va a servir para darle confianza al profesional y seguridad en su vida de preparación como profesional.

Los test que se aplica dan una orientación o guía para despertar el interés vocacional y el amor a lo que va ejercer como profesión. Este procedimiento tiene relevancia porque permite al estudiante a tomar decisiones acertadas en concordancia con sus aptitudes, competencias y capacidades.

Según (Vidal \& Fernández, 2009), define en su investigación: Es un instrumento de auto orientación que conjuga tres variables importantes relacionadas con la elección de la carrera: intereses, aptitudes y rendimiento, entendido como esfuerzo eficaz (...) y plantea algunas teorías y modelos que aconsejan el desarrollo de los programas desde su adecuada implementación en las ofertas de los centros educativos. La universidad de los niños es un espacio para aprender a descubrir quién eres y quien quieres ser, a través de la promoción de proyectos de vida y orientación sobre las profesiones que existen en la Universidad de Guayaquil.

Es un viaje al conocimiento porque realizaras un recorrido por las diferentes facultades, conociendo las aulas, laboratorios, bibliotecas y otros espacios donde se forman los profesionales, y así inspirarte a ser un estudiante exitoso.

El proyecto de vida es un camino para alcanzar una meta, un plan que se traza la persona para conseguir lo que quiere ser en la vida. Para ello debes contestarte las siguientes preguntas: ¿Quién soy?, ¿Cómo soy?, ¿Quién quiero ser? Para responder ¿Qué debes hacer para lograrlo?

La ejecución de este proyecto obedece al trabajo coordinado que está realizando Gesdecon con los líderes comunitarios en el contexto de la firma del convenio marco entre Holcim y la U.G.

El sábado 15 de Julio, 60 niños de las diferentes comunidades de Chongón y de la parroquia rural Juan Gómez Rendón (Progreso) se trasladaron con expectativa y alegría al Centro de Docencia e Investigación para el Desarrollo Humano y el Buen Vivir (C.D.I.D.) establecimiento perteneciente a la Facultad de Psicología de la Universidad de Guayaquil- U.G. Al llegar los niños sentían curiosidad por saber qué actividades realizarían. Estudiantes y profesores de la facultad de psicología y medicina de la U.G. los recibieron con dinámicas a fin de crear un ambiente de confianza para tener un viaje al conocimiento.

Estudiantes de la Facultad de ciencias químicas realizaron experimentos de reacciones químicas al mezclar diferentes sustancias, lo que motivo a los niños hacia nuevos conocimientos científicos.

Universidad de los Niños es un proyecto que propicia el acercamiento de niños y jóvenes al conocimiento científico y filosófico que se produce en la universidad, a partir de talleres orientados por la pregunta, la experimentación, la experiencia, el juego, la reflexión y el dialogo, 
con la finalidad que los asistentes visualicen un futuro profesional exitoso en un mundo para todos. Este proyecto se inició en la Universidad Alemana de Tubinga en el año 2002 y en la actualidad más de 200 universidades de todo el mundo lo implementan.

Dentro de los estudios de los niños se considera una de la parte principal el de considerar a la universidad como un elemento democrático que puede llegar a establecer que las pautas de consideración están establecidas en una serie de informaciones en el mejor lugar que es el mundo del conocimiento.

\section{Objetivo general}

Aproximar a los niños al conocimiento funcional de cada Facultad de la Universidad de Guayaquil mediante las visitas continuas para elegir correctamente su carrera.

\section{Objetivos específicos}

1. Promocionar los proyectos de vida orientando sobre las profesiones a elegir para disminuir el margen de error de la elección individual.

2. Gestionar la imagen de la Universidad de Guayaquil presentando las virtudes de cada carrera facilitando los indicadores de desempeño de cada Escuela.

\section{Marco teórico}

Los proyectos de vinculación que se desarrollan en la Universidad de Guayaquil (UG), están orientado a un nuevo modelo de pertinencia a través de la gestión social del conocimiento y la transferencia tecnológica, establecida para resolver problemas y dar soluciones concretas, las problemáticas, las necesidades en el campo productivo, social, político y cultural de la Zona 5 y 8; además propone líneas estratégicas surgidas de las necesidades de los actores y el análisis del entorno. La creación del sistema de vinculación de la UG, este articulado con los actores productivos, sociales académicos, políticos y culturales, que orienten a la organización de los proyectos de investigación, al desarrollo e innovación. Las practicas pre profesionales, los servicios a la comunidad, la integración al sistema educativo, la integración de equipos interdisciplinarios y de grupos colaborativas para el desarrollo del aprendizaje de los estudiantes, tiene como finalidad el desarrollo de las capacidades emprendedoras y de innovación entre las instituciones de educación superior y los sectores productivos, sociales, culturales y políticos.

Estos proyectos articulados a los ejes estratégicos de desarrollo y a los objetivos del plan nacional del buen vivir, que se considera son:

Mejorar la Calidad de vida de la población, Fortalecer las capacidades y potencialidades de la ciudadanía, Construir espacios de encuentro común, Fortalecer la identidad nacional, las identidades diversas, la plurinacionalidad y la interculturalidad, Garantizar el trabajo digno en todas sus formas, Impulsar la transformación de la matriz productiva.

Según indica el (Plan Nacional para el Buen Vivir, 2017), menciona: 
El tercer eje, "Más sociedad, mejor Estado", promueve la participación ciudadana y la construcción de una nueva ética social basada en la transparencia y la solidaridad, un Estado cercano con servicios de calidad y calidez, así como la soberanía y la paz, posicionando estratégicamente al Ecuador en el mundo. (pág. 11)

El estado tiene el compromiso de brindar apoyo económico para poder ejecutar estos proyectos y solucionar problemas a nivel educativo, social y que se brinde oportunidades en especial a los estudiantes que están en estado de vulnerabilidad y con problemas educativos.

El conocimiento de los niños y jóvenes para saber qué hacen los profesionales de cada carrera, permite realizar una investigación para diagnosticar las nociones sobre inteligencia, aprendizaje, motivación y comprensión en el aprendizaje de lo que muestra cada facultad, iniciando y preguntando en su centro de estudios a los directivos, docentes y estudiantes; sobre ¿cómo aprenden los estudiantes? ¿Qué es ingeniería? La Importancia de la motivación para el aprendizaje y el desarrollo industrial ¿qué es la medicina general?

Es un viaje al conocimiento porque realizaras una visita por las diferentes facultades, conociendo las aulas, laboratorios, bibliotecas y otros espacios donde se forman los profesionales, y así inspirarte a ser un estudiante con nociones de cómo y porqué se eligió la carrera que se está estudiando.

Podemos mencionar (Plan Nacional del Buen Vivir, 2017): Planificar para "toda una vida" implica una visión integral e integradora para que nadie, a lo largo de toda su vida, quede fuera o se quede atrás. (pág. 13)

La educación, la formación y la preparación académica es un proyecto de vida que se planifica a través del tiempo y desde sus inicios de estudios, el niño tiene sus aspiraciones y sueños por cursar una carrera que realmente ayude a desarrollarse y cumplir sus metas y objetivos. La integración de los conocimientos sobre las diversidades de profesiones que oferta la Universidad de Guayaquil en el proyecto Universidad de los niños es una clara visión hacia el futuro y el niño conozca cuáles serán sus posibilidades es escoger una carrera que realmente se sienta satisfecho y convencido de estudiar.

Además en los últimos años ha adquirido la vinculación con la sociedad un auge importante porque se establece una relación entre la universidad y la sociedad y mediante estoy proyectos se complementa se detecta los problemas, amenazas que podría afectar a la población más sensible o vulnerable y se une para dar servicio comunitario, mediante proyectos de rehabilitación comunidad que proporciona ayuda de diferentes índoles.

Estas actividades académicas influyen en experiencias enriquecedoras en la formación y mejoramiento pedagógico para los alumnos y en la búsqueda de solución a diferentes problemas que se suscitan a nivel regional y del Ecuador.

La vinculación de las IES con los sectores productivos contribuye positivamente en la formación y actualización de alumnos y académicos; en la solución de problemas a nivel local, regional y nacional; en la formación pedagógica de la planta docente; en la innovación y mejora 
de los procesos que tienen lugar en ambas partes; y en la inserción efectiva en la vida cultural y comunitaria (De Armas, 2009)

Este proyecto se inició en la Universidad Alemana de Tubinga en el año 2002 y en la actualidad más de 200 universidades de todo el mundo lo implementan. es un proyecto que propicia el acercamiento de niños y jóvenes al conocimiento científico y filosófico que se produce en la universidad, a partir de talleres orientados por la pregunta, la experimentación, la experiencia, el juego, la reflexión y el dialogo, Estos talleres se realizaron en las diferentes facultades que participaron como filosofía, medicina, arte, sistemas, ingeniería química psicología, con la finalidad que los asistentes visualicen un futuro profesional exitoso en un mundo para todos.

(Plan Nacional del Buen Vivir, 2017)

"En la última década identificar aquello que nos resta por hacer, para lograr alcanzar una vida digna para todos y todas en el horizonte común del Buen Vivir” (pág. 26)

El proyecto de vinculación tiene un propósito fundamental es lograr insertar a las personas en actividades productivas que permita subsistir en esta sociedad injusta con pocas oportunidades, lo ideal es ofrecer oportunidades de trabajo y desarrollo económico que aporte mejores posibilidades de mejor calidad de vida, sin educación y formación es difícil conseguir oportunidades de trabajo que ayuden a cubrir necesidades fundamentales de vida.

Cada semana asistían los estudiantes de Chongon en el horario de $7 \mathrm{~h} 30$ hasta las $12 \mathrm{~h} 30$ que finalizaba el trabajo. Durante el trabajo desarrollo, previa a una planificación elaborada por los estudiantes vinculantes de la universidad y la asesoría de los docentes tutores se realizaba actividades lúdicas motivadoras que se ejecutaban con los estudiantes que venían a participar en el proyecto y de acuerdo al cronograma desarrollado por vinculación con la comunidad se procedía al cierre con un programa especial de cada facultad que participaba n de los niños y los estudiantes de la universidad. Estos espacios de diálogo y acercamiento de los futuros estudiantes de la Universidad de Guayaquil hicieron que los chicos se plantearan que carrera o qué futuro desean para ellos.

\section{Diagnóstico y problema}

Descripción de la situación actual del área de intervención del proyecto

Preocupados porque la educación tradicional no llena las expectativas al momento de decidir una profesión se inició este proyecto en la búsqueda de acercar a los pequeños a la ciencia y la investigación que se produce en las universidades y además poder explicarles las diferentes profesiones que se ofertan en la universidad. Es un espacio de acercamiento a los niños para poder descubrir el ahora y el después en el proyecto de vida de los niños, motivar la curiosidad, el pensamiento crítico y descubrir su vocación que en el futuro podrá estudiar, competencias y habilidades tecnológicas, comunicativas, la metacognición.

Nos respaldaremos el artículo 26 de la (Constitución del Ecuador, 2008), para nuestros niños: 
"La educación es un derecho de las personas a lo largo de su vida y un deber ineludible e inexcusable del Estado. Constituye un área prioritaria de la política pública y de la inversión estatal, garantía de la igualdad e inclusión social y condición indispensable para el buen vivir.” (pág. 16)

Es un clamor de toda la sociedad integrar al sistema educativo la educación como una prioridad del ser humano para cumplir con sus expectativas en su vida y su familia.

Las sociedades y contribuir a su desarrollo y perfeccionamiento en el devenir de su vida. Las exigencias actuales de los profesionales es convertirse en una persona con muchas capacidades,

El principio del modelo de la Universidad de los niños es despertar la curiosidad, independiente de no tener recursos o no estar aptos para estar en la universidad, lo que se busca es motivar mediante una corriente pedagógica cuatro principios claros: La pregunta, la conversación, el juego y la experimentación.

\section{Metodología}

La investigación muestra los elementos que inciden en la toma de decisiones de los niños y adolescentes, con la finalidad de llegar a establecer un nexo o una orientación vocacional al momento de decidir su carrera profesional con la universidad de Guayaquil.

La metodología que se aplica en la investigación es exploratoria, que da una visión global de la realidad de la investigación y es descriptiva porque, mediante diálogos narrativos permite observar la realidad de los estudiantes participantes.

\begin{tabular}{cccc}
\multicolumn{4}{c}{ Resultados } \\
\hline ITEM & FRECUENCIA & VALORACIÓN & PORCENTAJE \\
\hline $\mathbf{1}$ & 50 & $\mathrm{MB}$ & $83 \%$ \\
& 10 & $\mathrm{~B}$ & $17 \%$ \\
& 0 & $\mathrm{R}$ & \\
TOTAL & 0 & $\mathrm{M}$ & $100 \%$ \\
\hline
\end{tabular}

Elaborado: Todos los vinculantes- Fuente: Niños Participantes (Empresa Holcim)

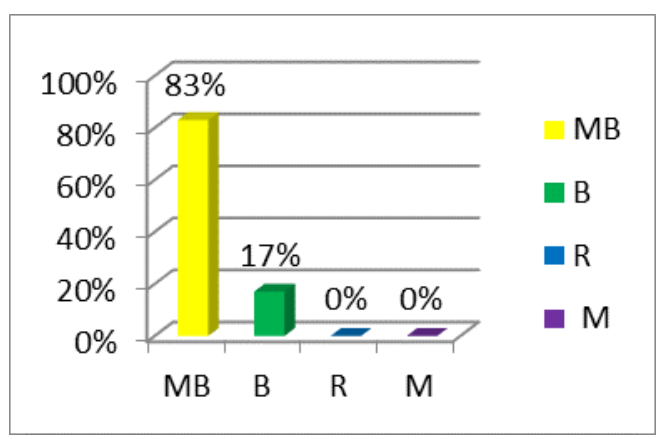

Elaborado: Todos los vinculantes-Fuente: Niños Participantes (Empresa Holcim) 
Análisis Estadístico: El 83\% de los vinculantes tuvieron una muy buena experiencia dentro del programa universidad de los niños y el 17\% obtuvo una buena acogida en el programa de la universidad de los niños.

Pregunta 2 ¿Te gustaría estudiar en la UG?

\begin{tabular}{cccc}
\hline ITEM & FRECUENCIA & VALORACIÓN & PORCENTAJE \\
\hline $\mathbf{2}$ & 60 & SI & $100 \%$ \\
& 0 & NO & 0 \\
TOTAL & 60 & & $100 \%$ \\
\hline
\end{tabular}

Elaborado: Todos los vinculantes Fuente: Niños Participantes (Empresa Holcim)

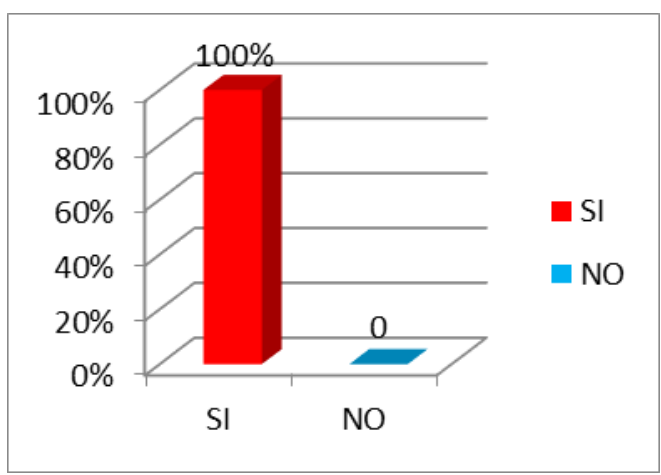

Elaborado: Todos los vinculantes

Fuente: Niños Participantes (Empresa Holcim)

Análisis Estadístico: El 100\% de los vinculantes están totalmente de acuerdo en estudiar en la U.G.

Pregunta 3: ¿En que facultad te gustaría estudiar en la UG?

\begin{tabular}{llll}
\hline ITEN & FRECUENCIA & VALORACIÓN & PORCENTAJE \\
\hline $\mathbf{3}$ & 12 & FILOSOFÍA & $20 \%$ \\
& 14 & MEDICINA & $23 \%$ \\
& 7 & MATEMÁTICAS & $12 \%$ \\
& 15 & FEDER & $25 \%$ \\
& 7 & PSICOLOGIA & $12 \%$ \\
& 3 & OTRA & $5 \%$ \\
& 2 & NINGUNA & $3 \%$ \\
TOTAL & 60 & & $100 \%$ \\
\hline
\end{tabular}

Elaborado: Todos los vinculantes

Fuente: Niños Participantes (Empresa Holcim) 


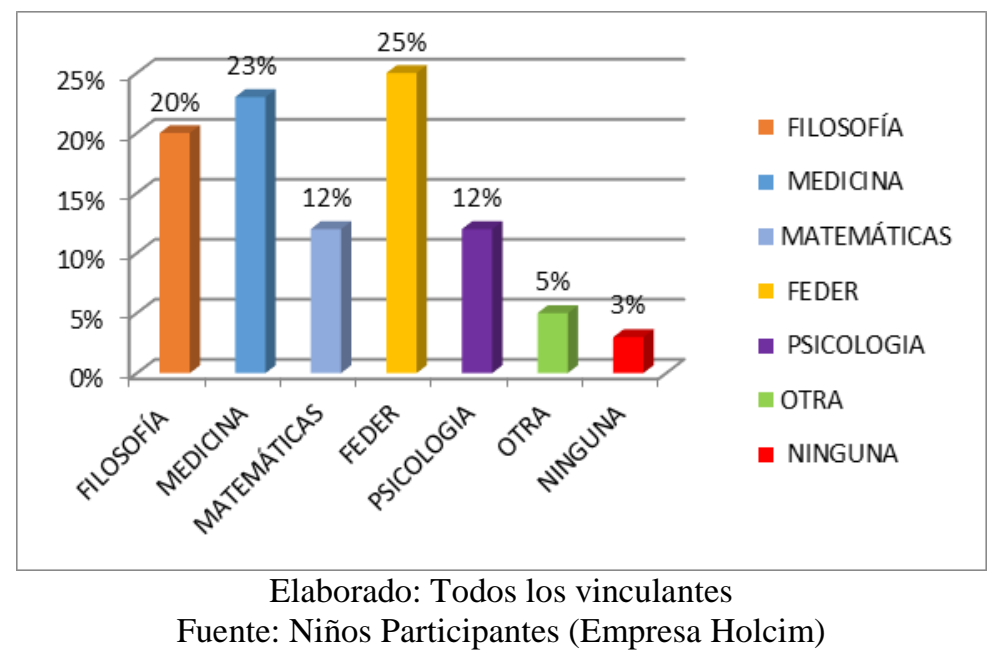

Análisis Estadístico: E $120 \%$ de los vinculantes les gustaría estudiar en la facultad de filosofía, el $23 \%$ en medicina, $12 \%$ en matemáticas, 25\% en FEDER, el12\% en psicología el 5\% en otra y el $3 \%$ en ninguna

\section{Pregunta 4}

¿Crees que sea importante para tu familia que ingreses en la UG?

\begin{tabular}{clll}
\hline ITEN & FRECUENCIA & VALORACIÓN & PORCENTAJE \\
\hline $\mathbf{4}$ & 57 & SI & $95 \%$ \\
& 3 & NO & $5 \%$ \\
TOTAL & 60 & & $100 \%$
\end{tabular}

Fuente: Niños Participantes (Empresa Holcim)

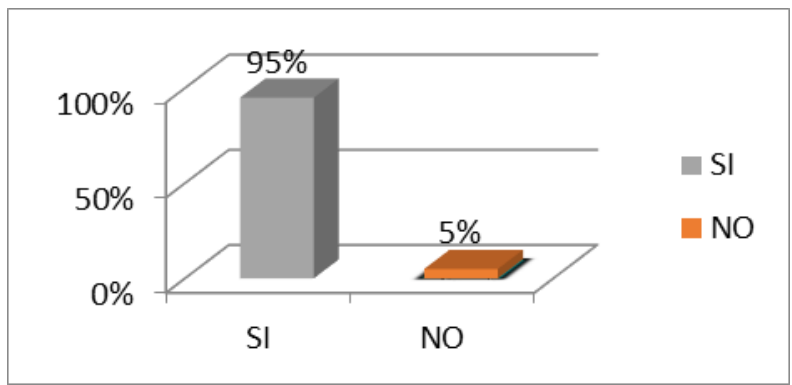

Elaborado: Todos los vinculantes

Fuente: Niños Participantes (Empresa Holcim)

Análisis Estadístico: el 95\% de los vinculantes están de acuerdos de que es importante para sus familiares estudiar en la universidad. El 5\% desacuerdo de que no es importante.

\section{Pregunta 5}

¿Te gustaría recibir más información de la UG? 


\begin{tabular}{llll}
\hline ITEM & FRECUENCIA & VALORACIÓN & PORCENTAJE \\
& \multicolumn{3}{c}{} \\
\hline $\mathbf{5}$ & 60 & SI & $100 \%$ \\
TOTAL & 0 & NO & $0 \%$ \\
\multicolumn{4}{c}{ Elaborado: Todos los vinculantes } \\
\multicolumn{4}{c}{ Fuente: Niños Participantes (Empresa Holcim) }
\end{tabular}

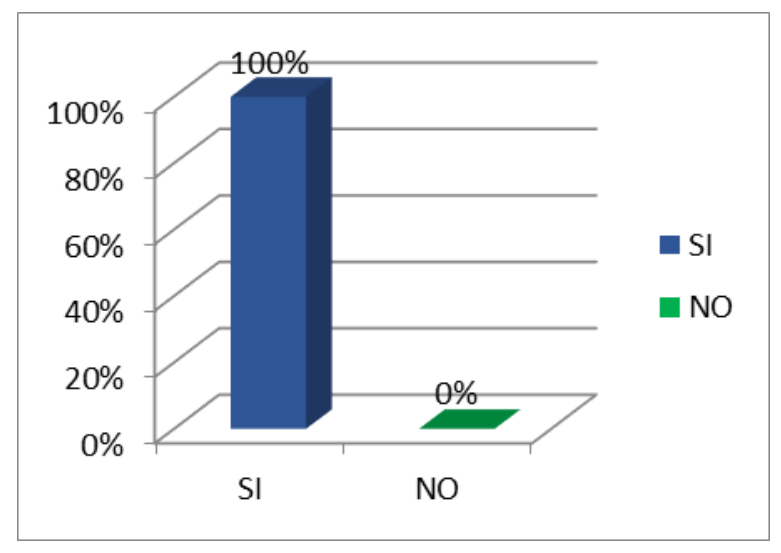

Elaborado: Todos los vinculantes Fuente: Niños Participantes (Empresa Holcim)

Análisis Estadístico: El 100\% de los vinculantes están de acuerdo en recibir más información sobre las facultades que les gustaría estudiar.

Art. 82.- Vinculación con la sociedad.- La vinculación con la sociedad hace referencia a los programas de educación continua, gestión de redes, cooperación y desarrollo, relaciones internacionales, difusión y distribución del saber que permitan la democratización del conocimiento y el desarrollo de la innovación social.

Las instituciones de educación superior deberán contar con un modelo de vinculación con la sociedad, que asegure la integración de las tres funciones sustantivas de la educación superior: docencia, investigación, y vinculación con la sociedad; para la gestión del conocimiento en función de sus dominios; líneas de investigación, oferta académica vigente y necesidades de la comunidad a nivel local, nacional y regional; respondiendo al principio de pertinencia.

Las instituciones de educación superior podrán crear instancias institucionales específicas para gestionar la vinculación con la sociedad, a fin de generar programas, proyectos específicos o intervenciones de interés público.

La vinculación se convierte en una relación de la universidad de Guayaquil con otros puntos de la provincia. La universidad porteña abre su mundo a los diferentes centros de vinculación. La universidad crea formas de recibimiento quizá una de las más complejas por lo que tiene que estar todo organizado, por el nuevo espacio en el que se trasladan los estudiantes, como por la poca direccionalidad que desconocen para llegar a un lugar.

Conclusiones 
En conclusión el proyecto de vinculación con la sociedad se justificó en los sectores de Chongón con estudiantes de diferentes edades y que durante la ejecución del mismo lograron tener una visión e imagen institucional de la Universidad de Guayaquil, como una entidad educativa de prestigio y que impacta en la comunidad. Las diferentes actividades que los niños participaron influenciaron en la satisfacción de los niños.

Es preciso que el proyecto de Gestión social del conocimiento, el líder la entidad que se firma el convenio haya una comunicación más directa a través de foros, reuniones de trabajo, mesas de trabajo para llegar a compromisos y mejoras del proyecto. Es de gran interés que todas las facultades participen en tal proyecto para su mejor difusión.

\section{Bibliografía}

Acosta, A. (2008). “El Buen Vivir, una oportunidad por construir”. Ecuador Debate 75: 33-47

Alonso, J. y P. Mosley (1999). La eficacia de la cooperación internacional al desarrollo: evaluación de la ayuda. Madrid: Editorial Cívitas.

Bretón. V. (2008). "Las contradicciones de las ONG de desarrollo rural. La trayectoria del FEPP en Chimborazo, 11981-2000”. En desarrollo rural y neoliberalismo, L. North y J. Cameron (Eds.): 181-203. Quito: UASB-Corporación editora nacional.

Cameron, J. (2008). "Democratización municipal y desarrollo rural en la sierra ecuatoriana". En Desarrollo rural y neoliberalismo, L. North y J. Cameron (Eds.): 205-230. Quito: UASBCorporación editora nacional.

CEPAL (2012). "Los países de renta media: un nuevo enfoque basado en brechas estructurales". Nota de la Secretaria. LC/G. 2532 (SES.34/11). Chiriboga, M. (1997). "Desafíos de la pequeña agricultura familiar frente a la globalización". En El desarrollo sostenible en el medio rural, Luciano Martínez (comp.). Quito: FLACSO.

Constitución del Ecuador. (22 de Agosto de 2008). Constitución del Ecuador. Obtenido de http://www.oas.org/juridico/PDFs/mesicic4_ecu_const.pdf:

Curiazi, R. (2014). Los proyectos solidarios de Salinas de Guaranda y su aporte para la construcción de "otra economía". http://repositorio.flacsoandes.edu.ec/handle/10469/7716

Egas Torres, D. (2015). El aporte de la cooperación internacional en el proceso de reforma de la justicia del Ecuador. http://repositorio.uasb.edu.ec/handle/10644/4827

Fundación ecuador proyectos integradores (2018). http://fe.org.ec/investigaciones/

Gallegos, Carlos (2016). Análisis del aporte de la implementación de un sistema integrado de información "ERP", en el mejoramiento de la gestión administrativa financiera de las empresas http://repositorio.uasb.edu.ec/handle/10644/4953 
Plan Nacional del Buen Vivir. (13 de Junio de 2017) Obtenido de https://observatorioplanificacion.cepal.org.cional\%20para\%20el\%20Buen\%20Vivir\%202 017-2021_0.pdf

Plan Nacional del Buen Vivir. (2017). Obtenido de https://observatorioplanificacion.cepal.org/sites/default/files/plan/files/Ecuador\%20Plan $\% 20 \mathrm{~N}$ cional\%20para\%20el\%20Buen\%20Vivir\%202017-2021_0.pdf

Plan Nacional para el Buen Vivir. (13 de Junio de 2017). Plan Nacional del Buen Vivi 2017-2021. Obtenido de https://observatorioplanificacion.cepal.org/sites/default/files/plan/files/Ecuador\%20Plan \%20Nacona1\%20para\%20el\%20Buen\%20Vivir\%202017-2021_0.pdf:

Sánchez Luna Victoria, J. Zaldívar Puig, M. (2016). Investigación científica y responsabilidad social: factores de impacto en las instituciones de educación superior del Ecuador Cofín $\begin{array}{lllll}\text { vol.10 no.2 } & \text { La } & \text { Habana } & \text { jul.-dic. }\end{array}$ http://scielo.sld.cu/scielo.php?script=sci_arttext\&pid=S2073-60612016000200011

Senescyt - Secretaría de Educación Superior (2014). Ciencia, Tecnología e Innovación Comunicamos Gobierno apuesta por la investigación científica https://www.educacionsuperior.gob.ec/gobierno-apuesta-por-la-investigacion-cientifica/ 